\title{
Low-Temperature Electroluminescence Excitation Mapping of Excitons and Trions in Short-Channel Monochiral Carbon Nanotube Devices
}

Marco Gaulke, Alexander Janissek, Naga Anirudh Peyyety, Imtiaz Alamgir, Adnan Riaz, Simone Dehm, Han Li, Uli Lemmer, Benjamin S. Flavel, Manfred M. Kappes, Frank Hennrich, Li Wei, Yuan Chen, Felix Pyatkov, and Ralph Krupke*

\begin{abstract}
Single walled carbon nanotubes as emerging quantum light sources may fill a technological gap in silicon photonics due to their potential use as near infrared, electrically driven, classical or nonclassical emitters. Unlike in photoluminescence, where nanotubes are excited with light, electrical excitation of single tubes is challenging and heavily influenced by device fabrication, architecture, and biasing conditions. Here we present electroluminescence spectroscopy data of ultra short channel devices made from $(9,8)$ carbon nanotubes emitting in the telecom band. Emissions are stable under current biasing, and no enhanced suppression is observed down
\end{abstract}
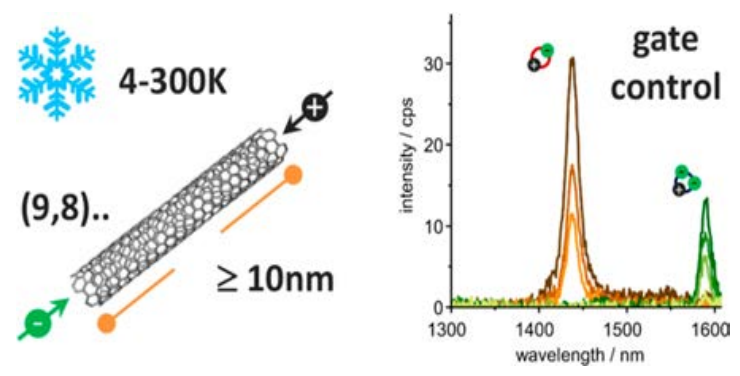
to $10 \mathrm{~nm}$ gap size. Low temperature electroluminescence spectroscopy data also reported exhibit cold emission and line widths down to $2 \mathrm{meV}$ at $4 \mathrm{~K}$. Electroluminescence excitation maps give evidence that carrier recombination is the mechanism for light generation in short channels. Excitonic and trionic emissions can be switched on and off by gate voltage, and corresponding emission efficiency maps were compiled. Insights are gained into the influence of acoustic phonons on the line width, absence of intensity saturation and exciton-exciton annihilation, environmental effects such as dielectric screening and strain on the emission wavelength, and conditions to suppress hysteresis and establish optimum operation conditions.

KEYWORDS: carbon nanotubes, light emission, electroluminescence, low temperature, devices

$\mathrm{T}$ he most valuable asset of single walled carbon nanotubes for photonics applications is their struc ture dependent optical transitions, which can be optically or electrically stimulated to emit light in the near infrared wavelength range. ${ }^{1,2}$ This, together with the compatibility of nanotubes to a range of biological, chemical, and CMOS processing methods, makes nanotubes highly attractive for applications as fluorescence markers in photo luminescence microscopy or as nanoscale emitters for on chip data transmission with light. ${ }^{3,4}$ For devices, the ability to scalably integrate specific nanotubes into complex architectures is essential, and the progress in synthesis and sorting of nanotubes and in selective placement has eventually materialized into electrically driven, ultrafast on chip light emitting devices that are susceptible to photonic engineering and at the verge of single photon emission. ${ }^{5,6}$ Short channel lengths are required for keeping the device footprint small and operation frequency high. However, if the channel length becomes comparable to the exciton diffusion length, the question arises at which point the emission will be quenched by nearby metal electrodes. ${ }^{7}$ So far electroluminescence from single tubes was explored for channel lengths down to a few hundreds of nanometers, ${ }^{8-12}$ or micrometers for aligned multitube devices and thin film devices. ${ }^{13-16}$ In this work we study emission from channels with an order of magnitude smaller gap size. For such small gaps the question arises whether emission will be broadened due to large electric fields and generation of hot carriers. This was reported in the pioneering works of the IBM group for micrometer size gaps $^{17,8}$ and is still unexplored for devices with a channel 
length of less than $100 \mathrm{~nm}$. Furthermore, whether electro luminescence in short channel devices would be driven by impact excitation ${ }^{18}$ or carrier recombination ${ }^{19}$ and how steady operating points for efficient light generation can be reached are open questions as well. In this work we have fabricated devices with a channel length down to 10 nanometers and recorded electroluminescence spectra under controlled biasing conditions over a wide temperature range. We studied preferentially monochiral $(9,8)$ nanotubes because they emit in the technologically relevant telecom band and can be synthesized through selective catalyst chemical vapor deposi tion (CVD) growth. ${ }^{20,21}$ At cryogenic conditions we observe excitonic and trionic emissions that are exceptionally stable and reproducible, appearing at well defined source-drain current and gate voltage. We have compiled electrolumines cence excitation maps that allow identifying optimum operating conditions, where emission lines are narrow and device efficiency is high. The enhanced stability is the result of a specific sample process flow, device biasing scheme, vacuum, and low temperature. We emphasize that the data constitute electroluminescence spectra recorded at cryogenic conditions. The results are important for understanding the mechanism behind light emission from short channel devices and to advance their potential use as on chip light sources.

\section{RESULTS AND DISCUSSION}

The $(9,8)$ nanotube devices with Pd source-drain electrodes were fabricated on $300 \mathrm{~nm} \mathrm{SiO}_{2} / \mathrm{p}$ doped $\mathrm{Si}$ substrates by electron beam lithography, metallization, and electric field assisted deposition of nanotubes (dielectrophoresis). The nanotubes were produced by selective catalyst CVD, dispersed in toluene by polymer wrapping, and purified and length sorted by gel filtration (details in the Methods section). Absorption spectra (Figure 1a) and photoluminescence excitation maps
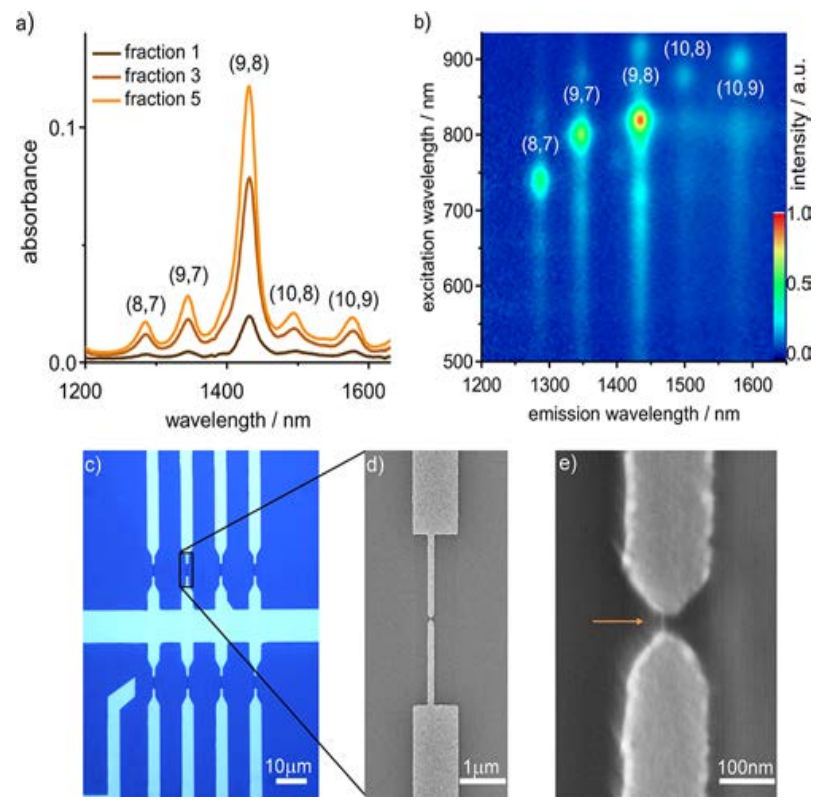

Figure 1. (a) Absorption spectra of length fractionated, polymer wrapped carbon nanotubes dispersed in toluene containing mainly the $(9,8)$ chirality. (b) Photoluminescence excitation map of fraction 5. (c, d) Optical image and scanning electron micrograph of the device layout. (e) Typical $(9,8)$ single tube contact formed after deposition from solution with dielectrophoresis. The position of the nanotube is indicated.
(Figure $1 \mathrm{~b}$ ) of excitonic transitions within the telecom band give evidence for a high content of $(9,8)$ nanotubes and the presence of $(8,7),(9,7),(10,8)$, and $(10,9)$ species in minor concentrations. To comply with the nominal charge transfer length for side contacted nanotubes, ${ }^{22}$ fractions of length sorted $(9,8)$ nanotubes were selected for depositions such that nanotubes were at least $200 \mathrm{~nm}$ longer than the distance between the source-drain electrodes. Simultaneous site selective deposition of single tubes from diluted nanotube dispersions onto multiple electrode pairs (Figure 1c), with channel lengths between 10 and $1000 \mathrm{~nm}$, was carried out by dielectrophoresis as described in the Methods section. A representative contact is shown in Figure 1d,e. The devices were electrically wired, mounted into an optical cryostat, and evacuated to $10^{-7}$ mbar. The cryostat is part of an optical microscopy and spectroscopy setup to image and analyze light emanating from devices with diffraction limited spatial resolution and $2 \mathrm{~nm}$ spectral resolution (1.2 meV @ 1440 $\mathrm{nm})$. Electroluminescence was measured in the application relevant telecom band from 1200 to $1610 \mathrm{~nm}$ and in the temperature range 4-300 K. All spectra were corrected by the relative spectral sensitivity of the setup. In the following we will report on electroluminescence spectra recorded from ultra short channel devices at cryogenic temperatures.

Figure $2 \mathrm{a}$ and $\mathrm{b}$ show $\mathrm{E}_{11}$ emission at room temperature from $(9,8)$ devices with 50 and $100 \mathrm{~nm}$ gaps, and the a)
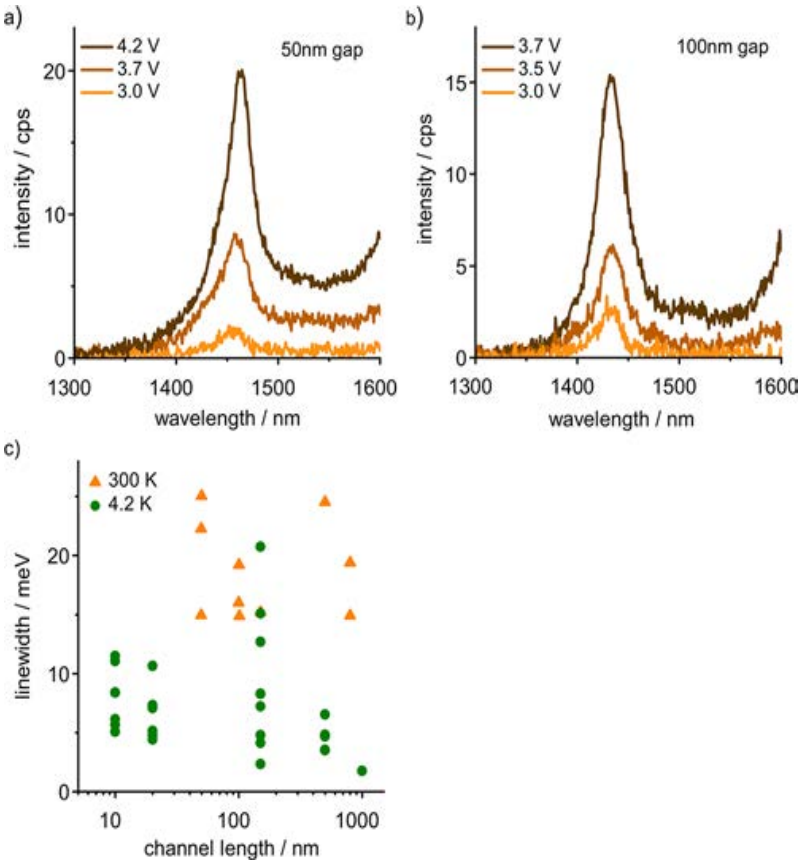

Figure 2. Room temperature electroluminescence spectra of $(9,8)$ short channel devices with a channel length of $50 \mathrm{~nm}(\mathrm{a})$ and 100 $\mathrm{nm}$ (b) under increasing source-drain bias voltage. (c) Full width at half maximum line width measured at 300 and $4 \mathrm{~K}$.

corresponding line widths for a number of devices with gap sizes between 10 and $1000 \mathrm{~nm}$ are shown in Figure 2c. The fact that we could measure electroluminescence down to 10 $\mathrm{nm}$ gap size was surprising, because from photoluminescence studies it is known that the exciton diffusion length in nanotubes at room temperature is on the order of hundreds of nanometers. ${ }^{23}$ It is also known that photoluminescence is quenched for fluorophores in direct contact with metallic 
surfaces. ${ }^{7}$ Therefore, if the device channel length is short compared to the nanotube exciton diffusion length, one would expect that the nonradiative recombination of excitons at the nearby source and drain metal contacts suppresses electro luminescence. In particular for ultra short channel devices with gaps between 10 and $50 \mathrm{~nm}$ we would have expected that electroluminescence becomes difficult to observe. On the other hand, the Strauf group recently reported plasmonic enhanced photoluminescence from nanotubes located on top of metallic bow tie antennas, having similar gap dimensions. ${ }^{24} \mathrm{~A}$ dielectric spacer was added on top of the metal structures to avoid quenching of photoluminescence. Such a layer is absent in our experiment. We might speculate that in our case the polymer shell around the nanotubes acts as a sufficiently thick dielectric spacer. On the basis of simulations of Wang et al., ${ }^{25}$ we can assume that the polymer shell has a thickness of $\sim 1 \mathrm{~nm}$, in agreement with the absence of a massive electrical tunneling barrier which appears for thicker polymer shells. For fluorophores, a $1 \mathrm{~nm}$ distance to a metal surface would be far too small to avoid quenching. ${ }^{7}$ On the other hand, for nanotubes on metal surfaces, Hong et al. have shown that the quenching distance $d$ is much smaller and well below $3 \mathrm{~nm}^{26}$ How can this be understood? Barnes has pointed out that the distance dependence of the decay rate follows $d^{-3}$ for nonradiative decay into bulk modes. ${ }^{27}$ This occurs if at the emission frequency electron scattering in the metal is strong, and it has been shown to be the case for fluorophores emitting in the visible. ${ }^{28}$ However, in the near infrared, where electron scattering is reduced and decay into bulk modes is suppressed, the decay into nonradiative surface plasmon polariton modes (SPP) prevails and a $d^{-4}$ dependence is expected. ${ }^{29}$ The exponent can be understood by the dipole-dipole Förster energy transfer rate, which depends inversely on the sixth power of the distance: ${ }^{28}$ For the case of a dipole above a metal, the problem is equivalent to one in which a point dipole transfers energy to a volume of point dipoles. The rate must be integrated over this volume, and the distance dependence is thereby reduced to cubic. From the same consideration one expects quartic distance dependence for transfer to a surface or thin film. Hence for near infrared active $(9,8)$ nanotubes it is understandable that the quenching distance is much smaller as for fluorophores emitting in the visible. On the other hand, electroluminescent light decaying into SPPs might also be recovered by the nanometer gap sized electrodes converting SPPs back to photons that couple to the far field.

Of course one could also envision a suppression of quenching by nanotube defects present in the gap region, in analogy to the defect induced reappearance of photolumines cence in ultrashort nanotubes. ${ }^{30}$ This is however unlikely to be the case here, since the electroluminescence data do not show the characteristic red shifted emission of an $\mathrm{sp}^{3}$ functionalized nanotube. ${ }^{30}$ From a technological point of view it is very promising that electroluminescence from carbon nanotubes is not bound to devices with large channel length. Also, the line width does not significantly broaden toward smaller gap size (Figure 2c), despite the very large induced electric field within the gap of up to $10^{8} \mathrm{~V} / \mathrm{m}^{31}$ Compared to the early electroluminescence work of the IBM group, ${ }^{8}$ the line width is more than an order of magnitude narrower and thermal ization of hot carriers appears to be sufficient to sustain cold emission even in ultra short channel devices.

We discuss now the temperature dependence of the excitonic emission of $(9,8)$ devices. Figure 3 a shows a set of
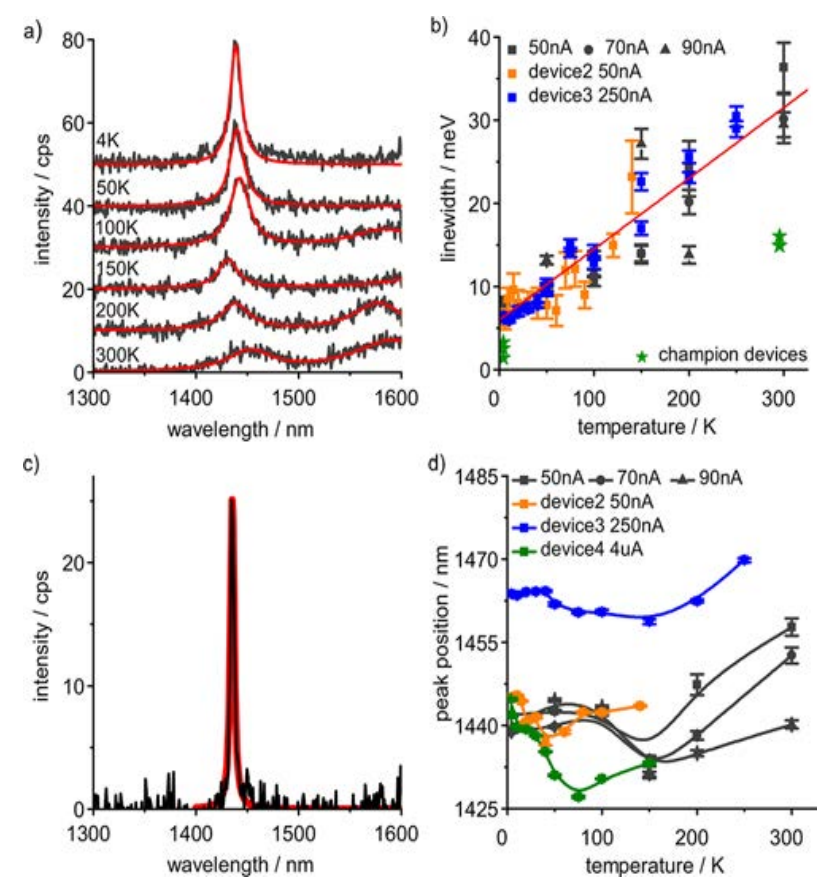

Figure 3. Temperature dependence of $(9,8)$ devices: (a) electro luminescence spectra, (b) line width, and (d) peak position. (c) Excitonic emission of a "champion device" with $2 \mathrm{meV}$ line width at $4 \mathrm{~K}$. Devices in (a) and (c) were measured at $70 \mathrm{nA}$, in (b) and (d) as indicated. Spectra were fitted with Lorentzian functions with uncertainties in line width and peak position given as error bars in (b) and (d). In (b) the data are fitted to the acoustic phonon mode coupling model described in the text.

electroluminescence spectra of a representative device measured between 4 and $300 \mathrm{~K}$. The $\mathrm{E}_{11}$ emission, visible at $\sim 1440 \mathrm{~nm}$, continuously narrows down from a $50 \mathrm{~nm}$ (30 $\mathrm{meV})$ line width at $300 \mathrm{~K}$ to a $5 \mathrm{~nm}(3 \mathrm{meV})$ line width at $4 \mathrm{~K}$. This dependency also holds for other $(9,8)$ devices at moderate current biasing $(<100 \mathrm{nA})$, as shown in Figure $3 \mathrm{~b}$. The line shape is Lorentzian throughout the entire temperature range. Since the line width is larger than the spectral resolution of our setup, we conclude that the homogeneous broadening is caused by exciton dephasing. ${ }^{32}$ Following the temperature dependent $(9,8)$ photoluminescence data of Yoshikawa et al., ${ }^{33}$ we assume that the temperature dependent part of the line width broadening is due to coupling to acoustic phonons. We fitted the line width $\Gamma$ in Figure $3 \mathrm{~b}$ to the expression $\Gamma=\Gamma_{0}+$ $A T+B\left(\exp \left(E_{\mathrm{ph}} / k_{\mathrm{B}} T\right)-1\right)^{-1} \cdot{ }^{34}$ Coefficients $A$ and $B$ are the exciton-phonon coupling constants for acoustic and optical phonons, respectively, and $E_{\mathrm{ph}}$ is the optical phonon energy. The temperature dependent electroluminescence line width data scatter around a straight line with $A \approx 0.085 \mathrm{meV} / \mathrm{K}$ and $B=0$, which indicates that the coupling to low frequency acoustic phonons determines the increase of the line width with temperature and that contributions of the high frequency modes are negligibly small, as observed by Yoshikawa et al. in photoluminescence. $^{33}$ Interestingly we see that the Lorentzian line width increases with the current bias and reaches up to $100 \mathrm{meV}$ at $300 \mathrm{nA}$ and at $4 \mathrm{~K}$ (Figure S1a,b). This is comparable to previous high bias electroluminescence meas urements of $(9,7)$ tubes, measured at room temperature, where exciton-exciton annihilation (EAA) was held responsible for a reduction of the lifetime with increasing current. ${ }^{35}$ However, we observe here that the emission intensity increases linearly with the current (Figure S1d) and not sublinear as expected for 
an EAA limited emission rate. ${ }^{36}$ Hence we conclude that EAA is not important here and consider dephasing by excitonexciton scattering as a source for current induced line broadening. ${ }^{37}$

Concerning the residual line width $\Gamma_{0}$ at $4 \mathrm{~K}$, we have measured on average between 3 and $5 \mathrm{meV}$ with champion devices down to $2 \mathrm{meV}$ (Figure $3 \mathrm{~b}, \mathrm{c}$ ). The $(9,8)$ line width at $4 \mathrm{~K}$ obtained by electroluminescence is therefore comparable to $(9,8)$ photoluminescence data. ${ }^{33,38}$ In some cases, albeit for other chiralities, photoluminescence line width was reported to reach $1 \mathrm{~nm}$ or below, with significant variations from tube to tube. ${ }^{39,40}$ These works indicate a sensitivity of the line width to the confinement of the low energy acoustic phonon modes and to exciton localization. ${ }^{41,40}$ Variations in the environment can easily induce these effects, and device to device variation of $\Gamma$ and of the EL peak positions is likely a result of limited precision in the directed placement of nanotubes, variations in the ordering of the polymer around the nanotube, and insufficient interface engineering on the atomic scale. Likewise we observe variations in the temperature dependence of the $\mathrm{E}_{11}$ peak position from device to device and with current (Figure 3d). Concerning the peak position, we observe for low currents a red shift up to $17 \mathrm{~nm}(10 \mathrm{meV})$ with increasing temperature from 4 to $300 \mathrm{~K}$. Sign and magnitude of the shift match with the expected temperature dependence of the nanotube bandgap. ${ }^{42}$ We also observe a blue shift at intermediate temperatures, which we may interpret as strain induced as reported for polymer wrapped $\nu=(n-m) \bmod 3=$ 1 nanotubes. ${ }^{43}$ The accumulation and the release of interfacial strain probably vary from device to device and may explain why blue shifts do not occur at identical temperatures. Unfortunately we have no data of other tube types to support this model. Also, we cannot exclude the effects of water that might accumulate inside the nanotubes even when filled with toluene from the processing. The solubility limit of water in toluene is $30 \mathrm{mmol} / \mathrm{L}$ at ambient conditions, ${ }^{44}$ and the nanotubes have open ends and are immediately exposed to any water layer that forms on the device surface. Still it remains unresolved whether phase transitions in the orientation of water dipoles contribute to the observed wavelength shifts. Molecular dynamics simulations show that the alignment of the dipoles and the freezing temperature is diameter dependent ${ }^{45,46}$ and occurs for suspended $(9,8)$ nanotubes between 250 and $290 \mathrm{~K}$. Since the high frequency permittivity of frozen water is smaller than liquid water, the dielectric screening changes accordingly. ${ }^{46,47}$ Hence the red shift in the upper temperature range might also be caused by melting of ice inside the carbon nanotube. Finally blue shifts could also be a signature of a transition from a localized state to free exciton state.

We report now on the influence of the back gate voltage on the emission characteristics in short channel devices. Figures 4a shows that for moderate current levels $(<100 \mathrm{nA})$ the excitonic emission can be completely suppressed when switching from $-2 \mathrm{~V}$ gate voltage to $+4 \mathrm{~V}$. At the same time a peak emerges at $\sim 1585 \mathrm{~nm}$, which for a fixed current bias can be gradually switched on to a peak intensity comparable to the excitonic peak (Figure $4 \mathrm{~b}$ ). This behavior is typical and shown here for two $(9,8)$ devices. We have measured similar dependencies also for other chiralities and determined the energy difference $\Delta E$ between the excitonic emissions and the corresponding red shifted emissions. For a selection of spectra from other chiralities we refer to Figure S4. Figure $4 c$ shows
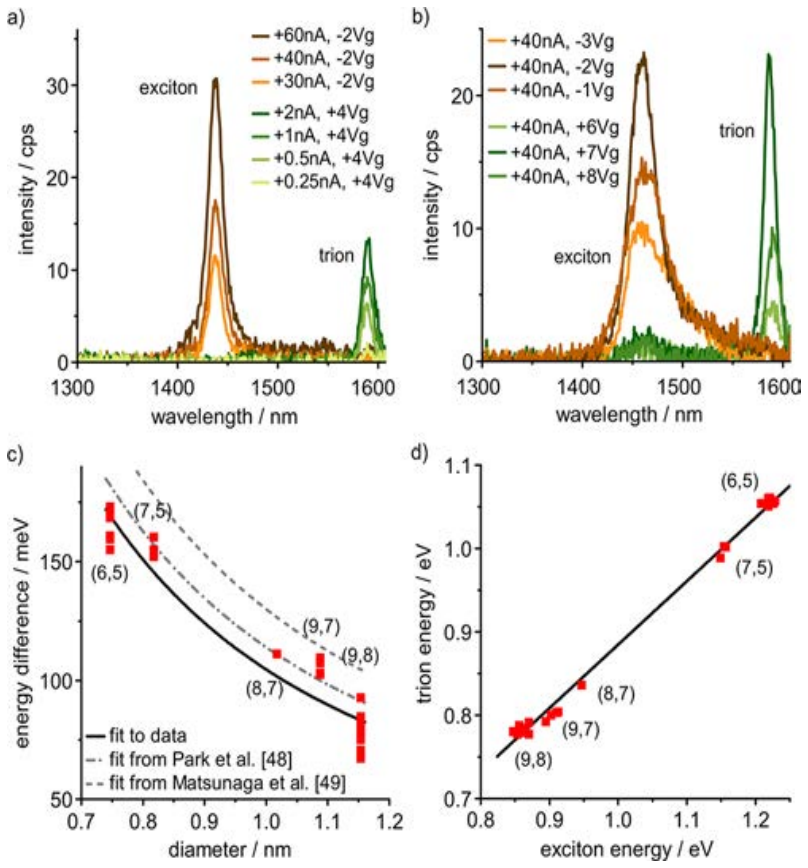

Figure 4. Gate controlled switching between excitonic and trionic emission from $(9,8)$ devices: (a) spectra acquired at discrete gate voltage $\left(V_{\mathrm{g}}\right)$ and increasing source-drain current and (b) increasing gate voltage and fixed source-drain current. (c) Energy difference between excitonic and trionic emission versus nanotube diameter. (d) Trion emission energy against exciton emission energy measured for several $(n, m)$ devices. In $(c)$ the dark line is a fit of the electroluminescence data to the sum of the diameter dependent trion binding energy and single-triplet exciton exchange splitting, in comparison to results from photolumines cence data shown in gray. Details to the fits are given in the text. All data are taken at $4.2 \mathrm{~K}$.

that the energy difference decreases with the nanotube diameter $d$ and fits to $\Delta E=A / d+B / d^{2}$ with $A=40 \pm 10$ $\mathrm{meV} / \mathrm{nm}$ and $B=65 \pm 9 \mathrm{meV} / \mathrm{nm}^{2}$. The data reproduces nicely the energy difference between excitons and trions observed by Park et al. in the photoluminescence of electrochemically doped nanotube films ${ }^{48}$ and by Jakubka et al. in the electroluminescence of thin film devices. ${ }^{15}$ We are therefore confident that the red shifted emission in the electroluminescence spectra of short channel devices under positive gate voltage stems from the recombination of trions. Trions in nanotubes were reported by Matsunaga et al. in the photoluminescence of hole doped nanotubes in solution, ${ }^{49}$ explaining that the energy difference observed between the excitonic and trionic emission equals the sum of the trion binding energy $\left(\propto d^{-1}\right)$ and the single-triplet exciton exchange splitting $\left(\propto d^{-2}\right)$, in agreement with theory. ${ }^{50}$ Figure $4 \mathrm{c}$ shows a comparison of our data with the other works. We note that the binding energy and the exchange energy, and hence $A$ and $B$, depend on the dielectric environment of the nanotube, ${ }^{47}$ and slight variations between experiments are expected. Plotting the trion emission energy against the exciton emission energy (Figure 4d), we find a linear correlation as expected from the diameter dependence of the excitonic emission ${ }^{47}$ and the diameter dependence of the energy difference between the excitonic and trionic emission.

For applications it is important to have stable and reproducible operating points for excitonic or trionic emission, and it is necessary to describe the conditions and biasing 
schemes used in this work. A major source for drift and irreproducibility in short channel electroluminescence meas urements is related to hysteresis in the transconductance curves. Figure S3 shows a typical measurement taken at room temperature $(296 \mathrm{~K})$ and under vacuum $\left(10^{-7} \mathrm{mbar}\right)$. A hysteresis of 7-8 $\mathrm{V}$ is prominent and typical for as prepared short channel devices. In such cases it is impossible to obtain stable steady state current and emission at a fixed gate voltage, because the filling and depleting of trap states with time results in an effective time dependent gating of the nanotube. Upon moderate heating the device to $343 \mathrm{~K}$ for $120 \mathrm{~min}$ within the evacuated cryostat, we observe a significant reduction of the hysteresis to $\sim 2 \mathrm{~V}$, which reduces to below $1 \mathrm{~V}$ at $220 \mathrm{~K}$ and disappears below $100 \mathrm{~K}$ (Figure 5a). A more rigid evaluation of
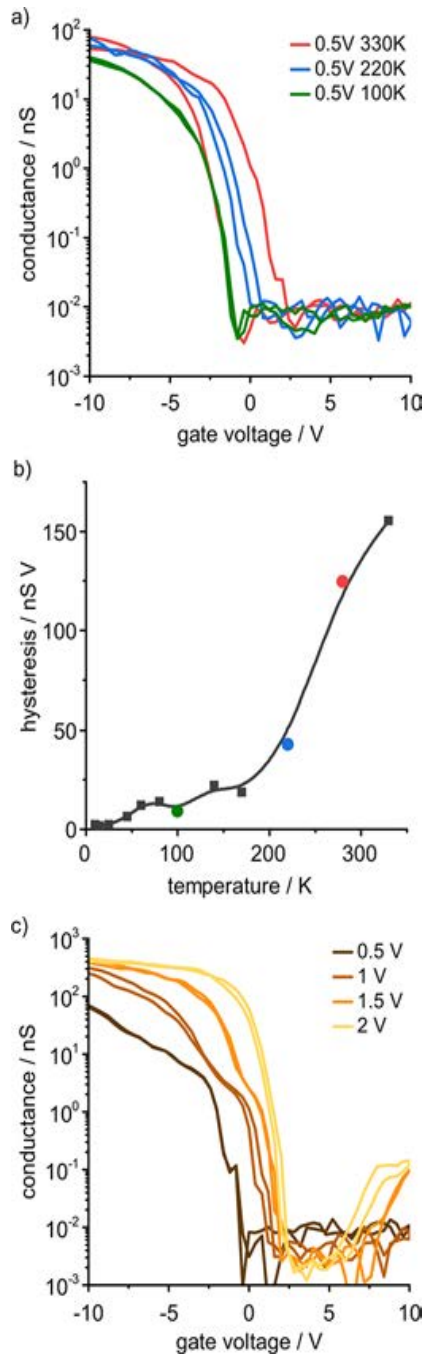

Figure 5. Electrical device characteristics: (a) Temperature dependence of the transconductance measured at fixed bias voltage. The hysteresis is defined as the area between the forward and backward sweeping curves and plotted in (b). (c) Trans conductance curves at $4.2 \mathrm{~K}$ with increasing voltage bias showing negligible hysteresis.

hysteresis is to integrate the area between the forward and backward sweeps of the transconductance curves, which shows that a full suppression of hysteresis occurs only at $4 \mathrm{~K}$ (Figure $5 \mathrm{~b}$ ). Water related trap states are probably the major source of hysteresis since moderate vacuum annealing already signifi cantly reduces hysteresis, and as discussed before, water, that may be encapsulated during device processing could play a role here as well. For stable light emission it is however also important to apply current biasing instead of voltage biasing. This is because the device resistance $R$ decreases with temperature $T(d R / d T<0)$, as seen from the temperature dependence of the ON state current (Figure 5a). Subsequently voltage biasing favors fluctuations in the electrical power dissipation and leads to unstable emission and thermal runaway. In contrast, current biasing stabilizes temperature, reduces power fluctuations, and leads to enhanced emission stability. Furthermore-and maybe most important-voltage biased nanotube devices often show unipolarity in the transconductance curves at low source-drain bias, with ambipolarity appearing only at larger bias, as shown in Figure 5c. This behavior is common for nanotube/metal contacts where the Schottky barrier for electrons is larger than for holes. It impedes finding optimum operating points for light emission since the regions of electron and hole conduction depend on both gate voltage and source-drain voltage. In contrast, by imposing source-drain current bias, the voltage required for compensating the different resistances in the respective $\mathrm{p}$ and $\mathrm{n}$ regions will be instantaneously applied by the source meter electronics. This leads to well defined gate voltage controlled $\mathrm{p}$ and $\mathrm{n}$ regions and pronounced ambipolarity.

Figure 6a shows the result of such current biasing for a $(9,8)$ device, where the measured voltage across the source-drain electrodes is plotted against the applied source-drain current and gate voltage. The $\mathrm{p}$ and $\mathrm{n}$ regions in the map are marked and separated by a region in which the voltage goes through a maximum at around $-10 \mathrm{~V}$ gate. This is the region with identical electron and hole currents, which is offset from the origin due to weak $\mathrm{n}$ doping. The asymmetry of the map is as discussed before due to nonidentical Schottky barriers for electrons and holes. During the measurement of the map, we have recorded simultaneously electroluminescence spectra. Spectra have been taken with an integration time of $10 \mathrm{~s}$ for each of the 42 steps in gate voltage and 5 steps in source-drain current, summing up to $35 \mathrm{~min}$ for the parameter space. The spectra were then integrated in wavelength sections corre sponding to the excitonic emission (1370-1500 nm) and the trionic emission (1500-1613 nm). The resulting excitonic and trionic excitation maps are shown in Figure $6 c$,d. Different regions can be identified in which predominantly excitons and trions are formed. Excitons are formed in the region with identical electron and hole currents (Figure 6c), which is evidence for light generation through carrier recombination and not impact excitation. This efficient mechanism of light generation has been observed previously in long channel and thin film devices, ${ }^{19,14}$ and this work now shows that carrier recombination is also dominating in short channel devices. The circumstances for trionic emission are somewhat more complicated, since the formation of trions requires a net charging of the nanotube channel, a condition that cannot be satisfied at charge neutrality. If we compare Figure $6 \mathrm{~d}$ with Figure $6 \mathrm{c}$ and Figure $6 \mathrm{a}$, we notice that the gate voltage range for trionic emission is shifted by $+10 \mathrm{~V}$ against the gate voltage for excitonic emission and, hence, is occurring in the region with excess electrons. We note that for $\mathrm{n}$ doped and $\mathrm{p}$ doped devices we observe the corresponding negatively charged trion $(\mathrm{T}-)$ and the positively charged trion $(\mathrm{T}+)$, respectively. Figure $\mathrm{S} 2$ shows an example of a p doped device with the $\mathrm{T}+$ emission occurring in the $p$ region (with excess of holes) at more negative gate voltages with respect to the excitonic 

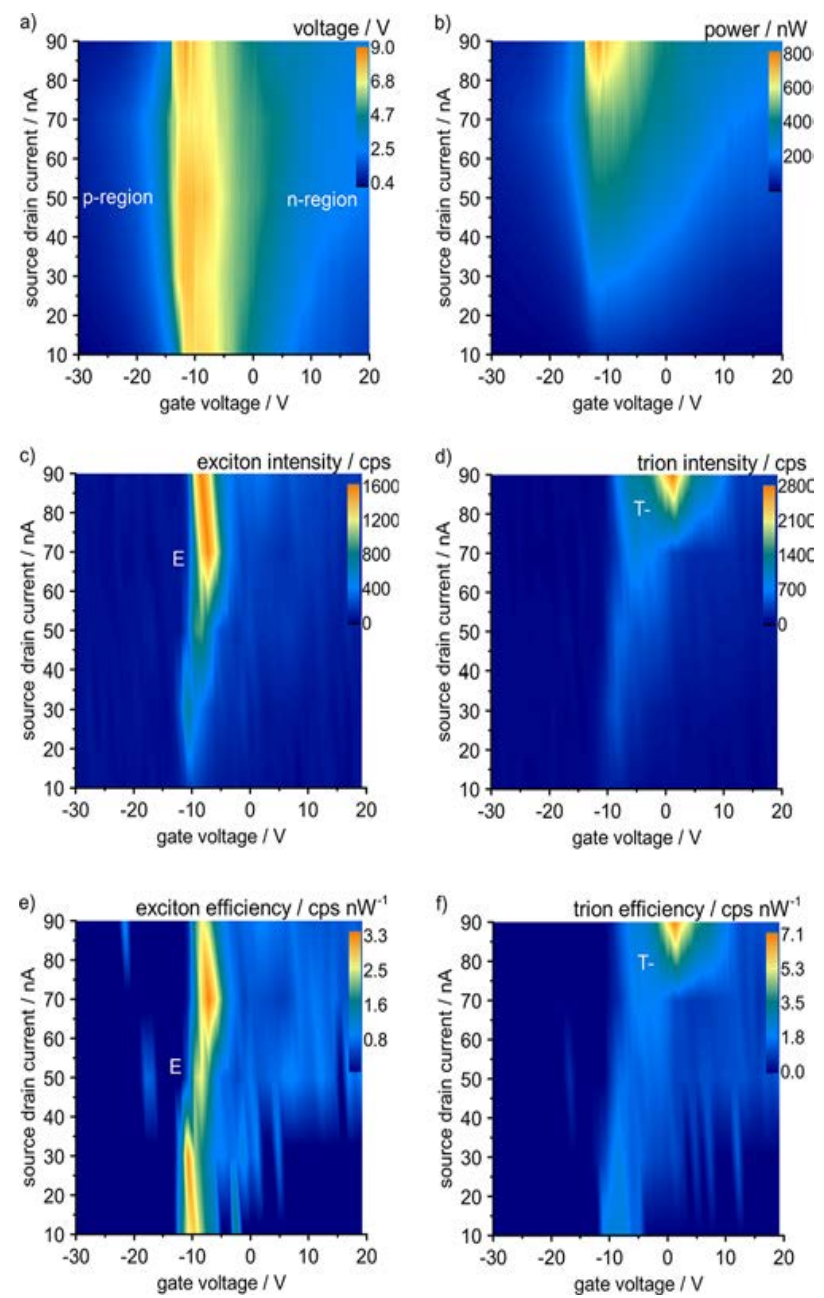

Figure 6. Current driven excitonic and trionic light emission from a weakly $\mathrm{n}$ doped $(9,8)$ device: (a) Source-drain voltage, (b) electrical power, and integrated $(c)$ exciton and $(d)$ trion intensity versus source-drain current and gate voltage (exiton range: 1370$1500 \mathrm{~nm}$; trion range: $1500-1613 \mathrm{~nm}$ ). Regions of electron $(\mathrm{n})$ and hole $(\mathrm{p})$ conduction are indicated. (e) Exciton and (f) trion efficiency in count rate/electrical power. The negatively charged trion $(\mathrm{T}-)$ appears in the $\mathbf{n}$ region, whereas the excitonic emission (E) appears close to charge neutrality between the $p$ and $n$ regions.

emission. We note that Liang et al. observed trionic emission at hole doping but no gate dependence. To identify conditions of enhanced emission efficiency, we have normalized the electroluminescence excitation maps in Figure $6 c$ and Figure $6 \mathrm{~d}$ with the electrical power dissipation map in Figure $6 \mathrm{~b}$. The obtained Figure $6 \mathrm{e}, \mathrm{f}$ yield maps of relative power efficiency for excitonic and trionic emission given as count rate per electrical power. We observe that the efficiency for excitonic emission peaks around $-10 \mathrm{~V}$ gate and is rather independent from the current, whereas the trionic emission at $+10 \mathrm{~V}$ gate becomes efficient only at larger current bias. We are lacking an explanation for this observation, but as shown in Figure S2 and Figure $4 \mathrm{a}, \mathrm{b}$ there are also examples where trion emission occurs already at very low current bias.

We also determined the electroluminescence quantum efficiency $\eta_{\text {ELQE }}=N_{\text {photons }} / N_{\text {charges }}$, the ratio of emitted photons and charges passing through the nanotube, which requires knowing the sensitivity of the setup to photons emitted by the nanotube. We approached the problem by calculating analytically the radiation pattern of an emitter on a layered substrate $^{51}$ and the fraction of emitted photons collected by the microscope objective and determined experimentally the factor that converts detector count rate into photon flux. The product of both yields the total setup efficiency $\eta_{\text {setup }}$ and converts detector count rate to photon flux for the specific experimental setup with a nanotube on a $300 \mathrm{~nm} \mathrm{SiO} / 2 \mathrm{Si}$ substrate. The spectra shown here were already corrected for the relative spectral sensitivity of the setup, and for $\lambda>1300$ $\mathrm{nm}$ the conversion into photon flux is then achieved by division with the factor $\eta_{\text {setup }}=8.7 \times 10^{-5}$ counts $\cdot \mathrm{s}^{-1} /$ photons . $\mathrm{s}^{-1}$. Details and procedures are described in the Supporting Information. We can directly calculate $\eta_{\mathrm{ELQE}}$ for excitonic and trionic emission via

$$
\eta_{\mathrm{ELQE}}=\frac{N_{\text {photon }}}{N_{\text {charges }}}=\frac{\text { intensity }(\mathrm{cps})}{\text { current }(\mathrm{A})} \frac{2 e}{\eta_{\text {setup }}}
$$

With $\eta_{\mathrm{ELQE}}=3 \times 10^{-6}$. intensity $[\mathrm{cps}] /$ current $[\mathrm{nA}]$ we obtain for devices at the optimum operating point $\eta_{\mathrm{ELQE}}=5 \times 10^{-4}$, which reproduces the earlier result from waveguide coupled $(9,7)$ nanotube emitters having a gap size of $1000 \mathrm{~nm}^{5}$ and implies that for $\eta_{\mathrm{ELQE}}$ the channel length does not play much of a role.

\section{CONCLUSION}

In summary, we have shown that electroluminescence in ultra short channel devices is not weaker than in long channel devices, likely due to relaxed quenching distance constraints in the near infrared. We have also realized on/off switching of excitonic and trionic emission by the gate voltage. Such control over the emission is important for the development of reliable and stable on chip light sources with narrow line emission in the telecom band. The cryogenic electroluminescence spec troscopy data shown in this work gives insights into the mechanism and the limitations of electrically induced light emission: The temperature dependence of the electro luminescence line width is in line with exciton dephasing caused by low energy acoustic phonons, whereas the homogeneous line broadening with increasing current at constant efficiency indicates dephasing by exciton-exciton scattering. The observed line width of $\sim 2 \mathrm{meV}$ at $4 \mathrm{~K}$ also shows that cold electroluminescence prevails even in short channel devices, which is promising for applications. Current biasing enforces ambipolarity and leads to stable, gate voltage only controlled operating points for light emission. By recording electroluminescence excitation maps we could verify that light emission in short channel devices is generated by carrier recombination. For applications at room temperature it will be crucial to obtain a similar reproducible, hysteresis free electroluminescence as observed at low temperature after vacuum annealing.

\section{METHODS}

Device Fabrication. Devices were prepared from commercial substrates (Active Business Company), which consist of a boron doped silicon carrier wafer (resistance $\Omega<0.005 \mathrm{~cm}$ ) covered with $300 \mathrm{~nm}$ of thermal silicon oxide. The wafer was diced to $10 \times 10 \mathrm{~mm}^{2}$ to fit into the cryostat setup, and electrodes were defined by electron beam lithography (Leo 1530) with proximity correction. While the preparation of structures with channel length of 150-1000 nm involved standard e beam patterning, the ultra short channel devices down to $10 \mathrm{~nm}$ electrode gap size were fabricated as follows. Samples were cleaned with acetone, isopropanol, and oxygen plasma and spin 
coated with $30 \mathrm{~nm}$ thick positive resist poly(methyl methacrylate) (PMMA, $950 \mathrm{~K} \mathrm{1 \%}$ in Anisol). After e beam patterning the sample was developed in a solution of methyl isobutyl ketone (MIBK) and isopropanol $\left(1: 3\right.$, for $30 \mathrm{~s}$ at $\left.0{ }^{\circ} \mathrm{C}\right)$ and annealed on a hot plate for 60 $s$ at $90{ }^{\circ} \mathrm{C}$. A $5 \mathrm{~nm}$ layer of chromium and $25 \mathrm{~nm}$ of palladium were deposited by sputtering technique. The lift off procedure was performed in acetone under mild sonication.

Preparation of CNT Suspensions and CNT Deposition. Single walled carbon nanotubes (SWCNTs) were synthesized using $\mathrm{CoSO}_{4} / \mathrm{SiO}_{2}$ as a catalyst and $\mathrm{CO}$ as a carbon precursor as described in detail. ${ }^{52}$ The catalyst $(200 \mathrm{mg})$ loaded in a $1 \mathrm{in}$. tubular reactor was reduced under $\mathrm{H}_{2}$ flow (1 bar, $50 \mathrm{sccm}$ ), while the reactor temperature was increased to $540{ }^{\circ} \mathrm{C}$. Then, the reactor temperature was further increased to $780{ }^{\circ} \mathrm{C}$ under Ar flow (1 bar, $\left.50 \mathrm{sccm}\right)$. Afterward, the catalyst was exposed to CO $(6 \mathrm{bar}, 100 \mathrm{sccm})$ to initiate SWCNT growth for $1 \mathrm{~h}$. Raw SWCNT soot was obtained after dissolving $\mathrm{SiO}_{2}$ in the catalyst loaded with SWCNTs in $\mathrm{NaOH}(1 \mathrm{M})$ solution. The preparation of CNT suspensions used in this study is described in full detail in our previous works. ${ }^{53}$ For SWCNT suspensions $100 \mathrm{mg}$ of the raw SWCNT soot and $100 \mathrm{mg}$ of the polymer poly (9,9 di $n$ dodecylfluorenyl 2,7 diyl) (PODOF) (Sigma Aldrich) were mixed in $100 \mathrm{~mL}$ of toluene and subjected to a sonication treatment for $2 \mathrm{~h}$ by using a titanium sonotrode (Bandelin, $\sim 20 \%$ power). During sonication, the suspension was placed in a water circulation bath to aid cooling. After sonication, the suspension was then centrifuged for $2 \mathrm{~h}$ at $20000 \mathrm{~g}$. To generate the starting suspensions for size exclusion separation, the supernatant was concentrated to $\sim 5 \mathrm{~mL}$ by evaporating $\sim 95 \mathrm{~mL}$ of toluene. Semipreparative, size exclusion chromatography was performed using Toyopearl HW 75 resin (Tosoh) filled into a glass column having a $16 \mathrm{~mm}$ inner diameter and $20 \mathrm{~cm}$ length. After application of $5 \mathrm{~mL}$ of SWCNT starting suspension to the gel, the sample was flowed through the gel under gravity, resulting in a flow rate of $\sim 2$ $\mathrm{mL} / \mathrm{min}$ with toluene as eluent. Fractions were collected in $\sim 4 \mathrm{~mL}$ portions. UV-vis-NIR absorption spectra of the fractions were recorded on a Varian Cary 500 spectrophotometer. Photolumines cence maps were measured in the emission range of $\sim 900-1700 \mathrm{~nm}$ and excitation range of 500-950 $\mathrm{nm}$ (scanned in $3 \mathrm{~nm}$ steps) using a modified FTIR spectrometer (Bruker IFS66) equipped with a liquid nitrogen cooled Ge photodiode and a monochromatized excitation light source as described elsewhere. ${ }^{54}$ Toluene based suspensions contain low chirality semiconducting nanotubes with a diameter of 1-1.2 nm, dominated by $(9,8)$ CNTs. Individual CNTs were simultaneously deposited from solution onto multiple contact pairs by capacitive coupled ac dielectrophoresis. ${ }^{55}$ The suspension was diluted by a factor of $10-100$ in order to deposit individual tubes, and a 20 $\mu \mathrm{L}$ droplet was placed onto the device array. A bias between 0.1 and 2 $\mathrm{V}$ at frequencies between $100 \mathrm{kHz}$ and $1 \mathrm{MHz}$ was applied between the common drain electrode and the back gate using an Agilent 33250 function generator. After $5 \mathrm{~min}$ the sample was rinsed with toluene to get rid of the excess polymer and annealed for $1.5 \mathrm{~h}$ at $160^{\circ} \mathrm{C}$ in order to improve the contact conductivity. To confirm the deposition of individual CNTs, transport characteristics of the devices were measured at ambient conditions in a probe station with TRIAX probes using an Agilent $4155 \mathrm{C}$ semiconductor parameter analyzer. For current biasing we have used for the source and the drain electrode separate source measurement units (SMU) and operated the Agilent 4155C in constant current mode. The drain electrode has been set as reference for the gate voltage, which has been applied by a third SMU in constant voltage mode.

Electroluminescence Spectroscopy and Cryogenic Setup. Samples were mounted on a custom made sample holder into a 4$500 \mathrm{~K}$ helium flow, sample in vacuum high resolution microscopy cryostat system (MicrostatHiResII, Oxford). Chip contacts of up to eight devices were bonded onto palladium pads attached to this holder. In situ annealing at $60-70{ }^{\circ} \mathrm{C}$ was conducted via the integrated heater at pressures below $10^{-6} \mathrm{mbar}$, and the subsequent electroluminescence measurements were carried out without breaking vacuum. The cryostat has a $10 \mathrm{~mm}$ diameter optical access via a 0.5 $\mathrm{mm}$ thick quartz window, and the emitted light was collected with a
Zeiss LD Plan Neofluar objective $(40 \times / 0.6)$ of a customized Zeiss Axiotech Vario microscope and focused with an off axis parabolic mirror (Thorlabs MPD149 P01, Ag, $25.4 \mathrm{~mm}, \mathrm{f} / 4$ ) into an Acton SP 2360 (f/3.9) imaging spectrograph (Princeton Instruments) and dispersed via a $85 \mathrm{G} / \mathrm{mm}, 1.35 \mu \mathrm{m}$ blazed grating onto an InGaAs photodiode linear array (PyLoN IR Princeton Instruments) with 1024 pixels, sensitive from 950 to $1610 \mathrm{~nm}$. The absolute spectral sensitivity of the setup was calibrated as described in the Supporting Information. The cryostat is positioned with sub $\mu \mathrm{m}$ precision by a motorized $x y$ scanning stage (8MTF, Standa), and the working distance between objective and sample surface is adjusted by a high precision objective piezo scanner (P 721 PIFOC/E 665 piezo amplifier, Physics Instruments), which allowed a precise and stable positioning of the emitter. CNT devices mounted in the cryostat were driven by an Agilent 4155B semiconductor parameter analyzer.

\section{AUTHOR INFORMATION}

\section{Corresponding Author}

Ralph Krupke - Karlsruhe Institute of Technology,

Karlsruhe, Germany, and Technische Universität Darmstadt, Darmstadt, Germany;

Email: krupke@kit.edu

\section{Other Authors}

Marco Gaulke - Karlsruhe Institute of Technology,

Karlsruhe, Germany, and Technische Universität Darmstadt, Darmstadt, Germany

Alexander Janissek - Karlsruhe Institute of Technology, Karlsruhe, Germany, and Technische Universität Darmstadt, Darmstadt, Germany

Naga Anirudh Peyyety - Karlsruhe Institute of

Technology, Karlsruhe, Germany, and Technische Universität

Darmstadt, Darmstadt, Germany

Imtiaz Alamgir - Karlsruhe Institute of Technology,

Karlsruhe, Germany

Adnan Riaz - Karlsruhe Institute of Technology,

Karlsruhe, Germany, and Technische Universität Darmstadt,

Darmstadt, Germany

Simone Dehm - Karlsruhe Institute of Technology,

Karlsruhe, Germany

Han Li - Karlsruhe Institute of Technology, Karlsruhe, Germany

Uli Lemmer - Karlsruhe Institute of Technology,

Karlsruhe, Germany;

Benjamin S. Flavel - Karlsruhe Institute of Technology, Karlsruhe, Germany;

Manfred M. Kappes - Karlsruhe Institute of Technology, Karlsruhe, Germany; 


\author{
Frank Hennrich - Karlsruhe Institute of Technology, \\ Karlsruhe, Germany \\ Li Wei - The University of Sydney, Sydney, Australia; \\ Yuan Chen - The University of Sydney, Sydney, \\ Australia; \\ Felix Pyatkov - Karlsruhe Institute of Technology, \\ Karlsruhe, Germany
}

\section{Author Contributions}

The experiments were conceived and designed by R.K., M.G., and F.P. The nanotube raw material was provided by L.W. and Y.C., purified by F.H., H.L., and B.F., and length fractionated by F.H. and M.M.K. Devices were fabricated by M.G., A.J., and S.D. The low temperature electroluminescence setup was built by M.G. with input from I.A., U.L., F.P., and R.K. Electroluminescence measurement were performed by M.G. and A.J. with input from F.P. and A.R. Simulations were performed by N.P. and R.K. The manuscript was written by R.K., M.G., and F.P. with input from all coauthors.

\section{Notes}

The authors declare no competing financial interest.

\section{ACKNOWLEDGMENTS}

A.R., F.P., and R.K. acknowledge funding by the Volkswagen Foundation. N.P., H.L., and B.F. acknowledge funding by the Deutsche Forschungsgemeinschaft (DFG). M.G., F.H., M.M.K., B.F., U.L., F.P., and R.K. acknowledge support by the Helmholtz Society through the program Science and Technology of Nanosystems (STN) and by the Karlsruhe Nano Micro Facility (KNMF). R.K. acknowledges support by Lukas Novotny for simulating dipole emission, Achim Hartschuh for insights into SPP modes, and Yoshikazu Homma for valuable information about environmental effects on carbon nanotubes.

\section{REFERENCES}

(1) Jorio, A., Dresselhaus, G., Dresselhaus, M. S., Eds. Carbon Nanotubes; Topics in Applied Physics Vol. 111; Springer: Berlin, Heidelberg, 2008.

(2) Avouris, P.; Freitag, M.; Perebeinos, V. Carbon Nanotube Photonics and Optoelectronics. Nat. Photonics 2008, 2, 341-350.

(3) Welsher, K.; Sherlock, S. P.; Dai, H. Deep Tissue Anatomical Imaging of Mice Using Carbon Nanotube Fluorophores in the Second Near Infrared Window. Proc. Natl. Acad. Sci. U. S. A. 2011, 108, 8943-8948.

(4) He, X.; Htoon, H.; Doorn, S. K.; Pernice, W. H. P.; Pyatkov, F.; Krupke, R.; Jeantet, A.; Chassagneux, Y.; Voisin, C. Carbon Nanotubes as Emerging Quantum Light Sources. Nat. Mater. 2018, 17, 663-670.

(5) Khasminskaya, S.; Pyatkov, F.; Słowik, K.; Ferrari, S.; Kahl, O.; Kovalyuk, V.; Rath, P.; Vetter, A.; Hennrich, F.; Kappes, M. M.; Gol'tsman, G.; Korneev, A.; Rockstuhl, C.; Krupke, R.; Pernice, W. H. P. Fully Integrated Quantum Photonic Circuit with an Electrically Driven Light Source. Nat. Photonics 2016, 10, 727-732.

(6) Pyatkov, F.; Fütterling, V.; Khasminskaya, S.; Flavel, B. S.; Hennrich, F.; Kappes, M. M.; Krupke, R.; Pernice, W. H. P. Cavity Enhanced Light Emission from Electrically Driven Carbon Nano tubes. Nat. Photonics 2016, 10, 420-427.

(7) Anger, P.; Bharadwaj, P.; Novotny, L. Enhancement and Quenching of Single Molecule Fluorescence. Phys. Rev. Lett. 2006, 96, 113002 .
(8) Freitag, M.; Perebeinos, V.; Chen, J.; Stein, A.; Tsang, J. C.; Misewich, J. A.; Martel, R.; Avouris, P. Hot Carrier Electro luminescence from a Single Carbon Nanotube. Nano Lett. 2004, 4, $1063-1066$.

(9) Pfeiffer, M. H. P.; Stürzl, N.; Marquardt, C. W.; Engel, M.; Dehm, S.; Hennrich, F.; Kappes, M. M.; Lemmer, U.; Krupke, R. Electroluminescence from Chirality Sorted $(9,7)$ Semiconducting Carbon Nanotube Devices. Opt. Express 2011, 19, A1184-A1189.

(10) Khasminskaya, S.; Pyatkov, F.; Flavel, B. S.; Pernice, W. H.; Krupke, R. Waveguide Integrated Light Emitting Carbon Nanotubes. Adv. Mater. 2014, 26, 3465-3472.

(11) Higashide, N.; Yoshida, M.; Uda, T.; Ishii, A.; Kato, Y. K. Cold Exciton Electroluminescence from Air Suspended Carbon Nanotube Split Gate Devices. Appl. Phys. Lett. 2017, 110, 191101.

(12) Wang, S.; Zeng, Q.; Yang, L.; Zhang, Z.; Wang, Z.; Pei, T.; Ding, L.; Liang, X.; Gao, M.; Li, Y.; Peng, L. M. High Performance Carbon Nanotube Light Emitting Diodes with Asymmetric Contacts. Nano Lett. 2011, 11, 23-29.

(13) Xie, X.; Islam, A. E.; Wahab, M. A.; Ye, L.; Ho, X.; Alam, M. A.; Rogers, J. A. Electroluminescence in Aligned Arrays of Single Wall Carbon Nanotubes with Asymmetric Contacts. ACS Nano 2012, 6, 7981-7988.

(14) Jakubka, F.; Backes, C.; Gannott, F.; Mundloch, U.; Hauke, F.; Hirsch, A.; Zaumseil, J. Mapping Charge Transport by Electro luminescence in Chirality Selected Carbon Nanotube Networks. ACS Nano 2013, 7, 7428-7435.

(15) Jakubka, F.; Grimm, S. B.; Zakharko, Y.; Gannott, F.; Zaumseil, J. Trion Electroluminescence from Semiconducting Carbon Nano tubes. ACS Nano 2014, 8, 8477-8486.

(16) Liang, S.; Ma, Z.; Wei, N.; Liu, H.; Wang, S.; Peng, L. M. Solid State Carbon Nanotube Device for Controllable Trion Electro luminescence Emission. Nanoscale 2016, 8, 6761-6769.

(17) Misewich, J. A.; Martel, R.; Avouris, Ph.; Tsang, J. C.; Heinze, S.; Tersoff, J. Electrically Induced Optical Emission from a Carbon Nanotube FET. Science 2003, 300, 783-786.

(18) Chen, J.; Perebeinos, V.; Freitag, M.; Tsang, J.; Fu, Q.; Liu, J.; Avouris, Ph. Bright Infrared Emission from Electrically Induced Excitons in Carbon Nanotubes. Science 2005, 310, 1171-1174.

(19) Freitag, M.; Chen, J.; Tersoff, J.; Tsang, J.; Fu, Q.; Liu, J.; Avouris, P. Mobile Ambipolar Domain in Carbon Nanotube Infrared Emitters. Phys. Rev. Lett. 2004, 93, 076803.

(20) Wang, H.; Wang, B.; Quek, X. Y.; Wei, L.; Zhao, J.; Li, L. J.; Chan Park, M. B.; Yang, Y.; Chen, Y. Selective Synthesis of $(9,8)$ Single Walled Carbon Nanotubes on Cobalt Incorporated TUD 1 Catalysts. J. Am. Chem. Soc. 2010, 132, 16747-16749.

(21) Wang, H.; Wei, L.; Ren, F.; Wang, Q.; Pfefferle, L. D.; Haller, G. L.; Chen, Y. Chiral Selective CoSO 4 /SiO 2 Catalyst for $(9,8)$ Single Walled Carbon Nanotube Growth. ACS Nano 2013, 7, 614626.

(22) Engel, M.; Steiner, M.; Sundaram, R. S.; Krupke, R.; Green, A. A.; Hersam, M. C.; Avouris, P. Spatially Resolved Electrostatic Potential and Photocurrent Generation in Carbon Nanotube Array Devices. ACS Nano 2012, 6, 7303-7310.

(23) Moritsubo, S.; Murai, T.; Shimada, T.; Murakami, Y.; Chiashi, S.; Maruyama, S.; Kato, Y. Exciton Diffusion in Air Suspended Single Walled Carbon Nanotubes. Phys. Rev. Lett. 2010, 104, 1-4.

(24) Luo, Y.; Ahmadi, E. D.; Shayan, K.; Ma, Y.; Mistry, K. S.; Zhang, C.; Hone, J.; Blackburn, J. L.; Strauf, S. Purcell Enhanced Quantum Yield from Carbon Nanotube Excitons Coupled to Plasmonic Nanocavities. Nat. Commun. 2017, 8, 1413.

(25) Wang, Y. J. Noncovalent Modification of Carbon Nanotubes by Conjugated Polymer: A Theoretical Study. Int. J. Quantum Chem. 2010, 111, 3897-3903.

(26) Hong, G.; Tabakman, S. M.; Welsher, K.; Wang, H.; Wang, X.; Dai, H. Metal Enhanced Fluorescence of Carbon Nanotubes. J. Am. Chem. Soc. 2010, 132, 15920-15923.

(27) Barnes, W. L. Fluorescence near Interfaces: The Role of Photonic Mode Density. J. Mod. Opt. 1998, 45, 661-699. 
(28) Waldeck, D. H.; Alivisatos, A. P.; Harris, C. B. Nonradiative Damping of Molecular Electronic Excited States by Metal Surfaces. Surf. Sci. 1985, 158, 103-125.

(29) Avouris, P.; Persson, B. N. J. Excited States at Metal Surfaces and Their Non Radiative Relaxation. J. Phys. Chem. 1984, 88, 837848.

(30) Danné, N.; Kim, M.; Godin, A. G.; Kwon, H.; Gao, Z.; Wu, X.; Hartmann, N. F.; Doorn, S. K.; Lounis, B.; Wang, Y.; Cognet, L. Ultrashort Carbon Nanotubes That Fluoresce Brightly in the Near Infrared. ACS Nano 2018, 12, 6059-6065.

(31) Perebeinos, V.; Avouris, P. Exciton Ionization, Franz-Keldysh, and Stark Effects in Carbon Nanotubes. Nano Lett. 2007, 7, 609-613.

(32) Klingshirn, C. F. Semiconductor Optics; Graduate Texts in Physics; Springer: Berlin, Heidelberg, 2012.

(33) Yoshikawa, K.; Matsunaga, R.; Matsuda, K.; Kanemitsu, Y. Mechanism of Exciton Dephasing in a Single Carbon Nanotube Studied by Photoluminescence Spectroscopy. Appl. Phys. Lett. 2009, 94, 093109.

(34) Karaiskaj, D.; Mascarenhas, A. Role of Electron Phonon Interactions and External Strain on the Electronic Properties of Semiconducting Carbon Nanotubes. Phys. Rev. B: Condens. Matter Mater. Phys. 2007, 75, 1-7.

(35) Pfeiffer, M. H. P.; Stürzl, N.; Marquardt, C. W.; Engel, M.; Dehm, S.; Hennrich, F.; Kappes, M. M.; Lemmer, U.; Krupke, R. Electroluminescence from Chirality Sorted $(9,7)$ Semiconducting Carbon Nanotube Devices. Opt. Express 2011, 19, A1184-A1189.

(36) Xiao, Y. F.; Nhan, T. Q.; Wilson, M. W. B.; Fraser, J. M. Saturation of the Photoluminescence at Few Exciton Levels in a Single Walled Carbon Nanotube under Ultrafast Excitation. Phys. Rev. Lett. 2010, 104, 017401.

(37) Nguyen, D. T.; Voisin, C.; Roussignol, P.; Roquelet, C.; Lauret, J. S.; Cassabois, G. Elastic Exciton Exciton Scattering in Photoexcited Carbon Nanotubes. Phys. Rev. Lett. 2011, 107, 127401.

(38) Lefebvre, J.; Finnie, P.; Homma, Y. Temperature Dependent Photoluminescence from Single Walled Carbon Nanotubes. Phys. Rev. B: Condens. Matter Mater. Phys. 2004, 70, 045419.

(39) Sarpkaya, I.; Zhang, Z.; Walden Newman, W.; Wang, X.; Hone, J.; Wong, C. W.; Strauf, S. Prolonged Spontaneous Emission and Dephasing of Localized Excitons in Air Bridged Carbon Nanotubes. Nat. Commun. 2013, 4, 1-8.

(40) Hofmann, M. S.; Noé, J.; Kneer, A.; Crochet, J. J.; Högele, A. Ubiquity of Exciton Localization in Cryogenic Carbon Nanotubes. Nano Lett. 2016, 16, 2958-2962.

(41) Vialla, F.; Chassagneux, Y.; Ferreira, R.; Roquelet, C.; Diederichs, C.; Cassabois, G.; Roussignol, P.; Lauret, J. S.; Voisin, C. Unifying the Low Temperature Photoluminescence Spectra of Carbon Nanotubes: The Role of Acoustic Phonon Confinement. Phys. Rev. Lett. 2014, 113, 057402.

(42) Capaz, R. B.; Spataru, C. D.; Tangney, P.; Cohen, M. L.; Louie, S. G. Temperature Dependence of the Band Gap of Semiconducting Carbon Nanotubes. Phys. Rev. Lett. 2005, 94, 036801.

(43) Karaiskaj, D.; Engtrakul, C.; McDonald, T.; Heben, M. J.; Mascarenhas, A. Intrinsic and Extrinsic Effects in the Temperature Dependent Photoluminescence of Semiconducting Carbon Nano tubes. Phys. Rev. Lett. 2006, 96, 4-7.

(44) Kirchnerová, J.; Cave, G. C. B. The Solubility of Water in Low Dielectric Solvents. Can. J. Chem. 1976, 54, 3909-3916.

(45) Ma, X.; Cambré, S.; Wenseleers, W.; Doorn, S. K.; Htoon, H. Quasiphase Transition in a Single File of Water Molecules Encapsulated in $(6,5)$ Carbon Nanotubes Observed by Temper ature Dependent Photoluminescence Spectroscopy. Phys. Rev. Lett. 2017, 118, 027402.

(46) Chiashi, S.; Saito, Y.; Kato, T.; Konabe, S.; Okada, S.; Yamamoto, T.; Homma, Y. Confinement Effect of Sub Nanometer Difference on Melting Point of Ice Nanotubes Measured by Photoluminescence Spectroscopy. ACS Nano 2019, 13, 1177-1182.

(47) Capaz, R. B.; Spataru, C. D.; Ismail Beigi, S.; Louie, S. G. Diameter and Chirality Dependence of Exciton Properties in Carbon
Nanotubes. Phys. Rev. B: Condens. Matter Mater. Phys. 2006, 74, 121401.

(48) Park, J. S.; Hirana, Y.; Mouri, S.; Miyauchi, Y.; Nakashima, N.; Matsuda, K. Observation of Negative and Positive Trions in the Electrochemically Carrier Doped Single Walled Carbon Nanotubes. J. Am. Chem. Soc. 2012, 134, 14461-14466.

(49) Matsunaga, R.; Matsuda, K.; Kanemitsu, Y. Observation of Charged Excitons in Hole Doped Carbon Nanotubes Using Photo luminescence and Absorption Spectroscopy. Phys. Rev. Lett. 2011, 106, 037404.

(50) Ando, T. Effects of Valley Mixing and Exchange on Excitons in Carbon Nanotubes with Aharonov-Bohm Flux. J. Phys. Soc. Jpn. 2006, 75, 024707.

(51) Novotny, L.; Hecht, B. Dipole Emission near Planar Interfaces. In Principles of Nano Optics; Cambridge University Press: Cambridge, 2012; pp 313-337.

(52) Wang, H.; Ren, F.; Liu, C.; Si, R.; Yu, D.; Pfefferle, L. D.; Haller, G. L.; Chen, Y. CoSO4/SiO2 Catalyst for Selective Synthesis of $(9,8)$ Single Walled Carbon Nanotubes: Effect of Catalyst Calcination. J. Catal. 2013, 300, 91-101.

(53) Hennrich, F.; Li, W.; Fischer, R.; Lebedkin, S.; Krupke, R.; Kappes, M. M. Length Sorted, Large Diameter, Polyfluorene Wrapped Semiconducting Single Walled Carbon Nanotubes for High Density, Short Channel Transistors. ACS Nano 2016, 10, $1888-1895$

(54) Lebedkin, S.; Hennrich, F.; Kiowski, O.; Kappes, M. M. Photophysics of Carbon Nanotubes in Organic Polymer Toluene Dispersions: Emission and Excitation Satellites and Relaxation Pathways. Phys. Rev. B: Condens. Matter Mater. Phys. 2008, 77, 1-8.

(55) Vijayaraghavan, A.; Blatt, S.; Weissenberger, D.; Oron Carl, M.; Hennrich, F.; Gerthsen, D.; Hahn, H.; Krupke, R.; Blatt, S.; Vijayaraghavan, A. Ultra Large Scale Directed Assembly of Single Walled Carbon Nanotube Devices. Nano Lett. 2007, 7, 1556-1560. 


\section{Repository KITopen}

Dies ist ein Postprint/begutachtetes Manuskript.

Empfohlene Zitierung:

Gaulke, M.; Janissek, A.; Peyyety, N. A.; Alamgir, I.; Riaz, A.; Dehm, S.; Li, H.; Lemmer, U.; Flavel, B. S.; Kappes, M. M.; Hennrich, F.; Wei, L.; Chen, Y.; Pyatkov, F.; Krupke, R. Low-Temperature Electroluminescence Excitation Mapping of Excitons and Trions in Short-Channel Monochiral Carbon Nanotube Devices.

2020. ACS nano, 14.

doi: $\underline{10.5445 / / \mathrm{R} / 1000117970}$

Zitierung der Originalveröffentlichung:

Gaulke, M.; Janissek, A.; Peyyety, N. A.; Alamgir, I.; Riaz, A.; Dehm, S.; Li, H.; Lemmer, U.; Flavel, B. S.; Kappes, M. M.; Hennrich, F.; Wei, L.; Chen, Y.; Pyatkov, F.; Krupke, R. Low-Temperature Electroluminescence Excitation Mapping of Excitons and Trions in Short-Channel Monochiral Carbon Nanotube Devices. 2020. ACS nano, 14 (3), 2709-2717.

doi:10.1021/acsnano.9b07207 